\title{
NOWHERE DENSE SETS AND REAL-VALUED FUNCTIONS WITH CLOSED GRAPHS
}

\author{
IVAN BACGS \\ Department of Mathematics \\ University of Alberta \\ Edmonton, A1berta T6G 2G1 \\ Canada
}

(Received Apri1 19, 1988 and in revised form May 10, 1988)

ABSTRACT. Closed and nowhere dense subsets which coincide with the points of discontinuity of real-valued functions with a closed graph on spaces which are not necessarily perfectly normal are investigated. Certain $G_{\delta}$ subsets of completely regular and normal spaces are characterized. It is also shown that there exists a countable connected Urysohn space $X$ with the property that no closed and nowhere dense subset of $X$ coincides with the points of discontinuity of a real-valued function on $X$ with a closed graph.

KEYWORDS AND PHRASES. Rea1-valued functions with closed graphs, nowhere dense sets, $G_{\delta}$ sets and non-perfectly normal spaces.

1980 AMS SUBJECT CLASSIFICATION CODES. 54C10, 54C30, $54 \mathrm{C} 50$.

1. INTRODUCTION.

Let $X$ and $Y$ denote topological spaces. Let $R$ denote the real line with the usual topology. A11 spaces w111 be assumed to be $T_{2}$. Let $f$ be a function from $X$ into $Y$ and denote the graph of $f$ by $G(f)=\{(x, f(x)) \in X \times Y \mid x \in X\}$. The graph of $f$ is closed if $G(f)$ is a closed subset of $X \times Y$. Let $D(f)=\{x \in X \mid f \quad$ is discontinuous at $x\}$.

It was shown by Thompson [1] that if $X$ is a Baire space and if a function $f: X+R$ has $G(f)$ closed, then $D(f) \quad 1 s$ a closed and nowhere dense subset of $X$. It was proven by Dobǒs [2] that, if $X$ is perfectly normal, $F \subset X$ is closed and of first category in $X$ if and only if there exists a function $f: X \rightarrow R$ such that $G(f)$ is closed and $D(f)=F$.

Let $X$ be a topological space which is not necessarily perfectly normal. In this note we will investigate those closed and nowhere dense subsets of $X$ which coincide with the points of discontinuity of a real-valued function with a closed graph on $X$. We will consider this problem when $X$ is elther a normal, a completely regular, a regular or a Urysohn space. 
2. PRELIMINARIES. In what follows we will make use of the following known results.

THEOREM 2.1. Let $X$ be a topological space. If $f: X \rightarrow K$ has a closed graph, then $D(f)$ is closed and of the first category in $X$ (Dobǒs [2]).

THEOREM 2.2. Let $\mathrm{f}: \mathrm{X} \rightarrow \mathrm{Y}$ be a function with a closed graph. If $\mathrm{K}$ is a compact subset of $Y$, then $f^{-1}(K)$ is a closed subset of $X$ (Hamlett and Herrington [3]).

PROPOSITION 2.3. If $\mathrm{h}: \mathrm{X} \rightarrow \mathrm{Y}$ is a continuous function and if $\mathrm{g}: \mathrm{Y} \rightarrow \mathrm{Z}$ is a function with a closed graph, then $f=g \circ h$ has a closed graph (Thompson [1]).

PROPOSITION 2.4. Let $X$ be a completely regular topological space. If $F$ is a compact $G_{\delta}$ subset of $X$, then there exists a continuous function $f: X+R$ such that $F=\{x \in X \mid f(x)=0\}$ (Gillman and Jerison [4], page 43).

If $V$ is a closed subset of $X$, throughout $\bar{V}$ and $V^{c}$ will be used to denote the closure of $V$ and the complement of $V$, respectively. $[a, b]$ will be used to denote a closed interval in $R$.

3. X IS NORMAL. We will characterize closed and nowhere dense $G_{\delta}$ subsets of a normal space $X$ in terms of the points of discontinuity of a real-valued function with a closed graph. We will first establish the following lemma.

LEMMA 3.1. Let $F \subset X$ be closed and nowhere dense in $X$ and let $f: X \rightarrow R$ be a function with $G(f)$ closed such that $D(f)=F$. If for each net $\left\{x_{\alpha} \mid \alpha \in I\right\}$ in $X-F$ which converges to $x \in F,\left\{f\left(x_{\alpha}\right) \mid \alpha \in I\right\}$ does not converge in $R$, then $F$ is $\mathrm{G}_{\delta}$.

PROOF: Case (1). Suppose $f$ is constant on $F$. Let $\varepsilon>0$. For each $x \in X$, let $V_{\varepsilon}(f(x))$ denote an open interval in $R$ of radius $\varepsilon$ and centered at $f(x)$. Select for each $x \in F$ an open neighbourhood $U(x)$ of $x$ such that $f(U(x)-F) \subset\left[V_{\varepsilon} f(x)\right]^{c}$. (This is possible, for otherwise given $v_{\varepsilon}(f(x)$ ), there would exist for each neighbourhood $U(x)$ of $x$ an element $y_{U} \in[U(x)-F]$ such that $f\left(y_{U}\right) \in V_{\varepsilon}(f(x))$. This would imply $y_{U}+x$ and $f\left(y_{U}\right)+f(x)$ which is impossible.) Put $U=\bigcup_{x \in F} U(x)$. Clearly $F \subset U$. Let $x \in F$ and put

$$
v_{n}=\left\{\left[f(x)-n, f(x)-\frac{1}{n}\right] \cup\left[f(x)+\frac{1}{n}, f(x)+n\right]\right\}, n=1,2, \ldots
$$

$V_{n}$ is a compact subset of $R$ for each $n$ and, by Theorem $2.2, f^{-1}\left(V_{n}\right)$ is closed in $X$ for each $n$. Put $G_{n}=\left[f^{-1}\left(V_{n}\right)\right]^{c} \cap U$ for $n=1,2, \ldots$ Since $f$ is constant on $F, F \subset\left[f^{-1}\left(V_{n}\right)\right]^{c}$ for each positive integer $n$. Let $y \in U-F$. It follows that $f(y) \epsilon\left[f(x)-n_{0}, f(x)-\frac{1}{n_{0}}\right] \cup\left[f(x)+\frac{1}{n_{0}}, f(x)+n_{0}\right]$ for $x \in F$ and for some integer $n_{0}$. Therefore $y \notin G_{n_{0}}$ and $y \notin \bigcap_{n=1}^{\infty} G_{n} \subset U$. Since $F \subset G_{n}$ for each $n$, it follows that $F=\bigcap_{n=1}^{\infty} G_{n}$ and $F$ is $G_{\delta}$, since $G_{n}$ is open for each $n$.

Case (ii). Suppose $f$ is not constant on $F$. Let $x_{1} \in F$ be fixed. Define $g: X+R$ by $g(x)=f\left(x_{1}\right)$ if $x \in F$ and $g(x)=f(x)$ otherwise. It follows that $G(g)$ is closed since $G(f)$ is closed and $g$ satisfies the hypothesis of the 
1emma. Now by Case (1), $F$ is $G_{\delta}$ and the lemma is established.

Hamlett and Herrington [3] gave an example (Example I.6.1) to show that if $X$ is the space of all ordinals less than or equal to the first uncountable ordinal, $\Omega$, then there does not exist a function $f: X+R$ with a closed graph such that $D(f)=\{\Omega\}$.

THEOREM 3.2. Let $X$ be a normal space and let $F \subset X$ be closed and nowhere dense in $X . \quad F$ is $G_{\delta}$ if and only if there exists a function $f: X \rightarrow R$ such that $G(f)$ is closed, $D(f)=F$ and for every net $\left\{x_{\alpha} \mid \alpha \in I\right\} \subset X-F$ which converges to $x \in F,\left\{f\left(x_{\alpha}\right) \mid \alpha \in I\right\}$ does not converge in $R$.

PROOF: Sufficiency follows from the proceeding lemma. To show necessity suppose $F$ is $G_{\delta}$. It follows that there exists a continuous function $h: X+R$ such that $\{x \in X \mid h(x)=0\}=F$ (Kuratowsk1 [5], page 134). Define $f: R \rightarrow R$ by $g(0)=0, g(x)=\frac{1}{x}$, otherwise. Put $f=g \circ h$. It follows from Proposition 2.3 that the graph of $f$ is closed. Clearly $f$ has the required properties.

In Example 4.1 it will be shown that 'normal' cannot be replaced by 'completely regular' in Theorem 3.2. First we will establish two lemmas which will be used in this example.

LEMMA 3.3. If $f: X+R$ has a closed graph and if $x \in D(f)$, where $D(f)$ is nowhere dense in $x$, then there exists a net $\left\{x_{\alpha} \mid \alpha \in I\right\} \subset[D(f)]^{c}$ such that $x_{\alpha} \rightarrow x$ and $\left\{f\left(x_{\alpha}\right) \mid \alpha \in I\right\}$ has no convergent subnet in $R$.

PROOF: Let $x \in D(f)$. There exists some net $\left\{y_{\alpha}\right\}$ in $x$ such that $y_{\alpha} \rightarrow x$ and $f\left(y_{\alpha}\right)$ has no convergent subnet in $R$. We may assume without loss of generality that $y_{\alpha} \in D(f)$ for all $\alpha$ and that $\left|f\left(y_{\alpha}\right)\right|>|f(x)|+1$ for all $\alpha$. For each open neighbourhood $U$ of $x$, select $y_{\alpha(U)} \in U$ and $x_{\alpha(U)} \in U \cap[D(f)]^{c}$ such that $\left|f\left(x_{\alpha(U)}\right)\right| \geq\left|f\left(y_{\alpha(U)}\right)\right|-\varepsilon$. Such an $x_{\alpha(U)}$ exists for each $U$ and each $y_{\alpha(U)}$ since $y_{\alpha(U)} \in D(f)$ which is nowhere dense in $x$, and, if $y_{\alpha(U)}$ is any net in $[D(f)]^{c}$ which converges to $y_{\alpha(U)}$, then either $\left.f_{\left(y_{\alpha(U)}\right)}\right) f_{\beta}\left(y_{\alpha(U)}\right)$ or $f\left(y_{\alpha(U)_{\beta}}\right)$ has no convergent subnet in R. Clearly $x_{\alpha(U)}+x$ and $f\left(x_{\alpha(U)}\right)$ does not converge to $f(x)$. Since the graph of $f$ is closed, it follows that $f\left(x_{\alpha(U)}\right)$ has no convergent subnet in $R$.

LEMMA 3.4. Let $f, g: X \rightarrow R$ be two functions with closed graphs. If there exists a dense subset $X_{1}$ of $X$ such that $f\left|X_{1}=g\right| X_{1}$, then $D(f)=D(g)$.

PROOF: Suppose $x \in D(f)$. By Lemma 3.3 there exists a net $\left\{x_{\alpha} \mid \alpha \in I\right\} \subset[D(f)]^{c}$ such that $x_{\alpha}+x$ and $f\left(x_{\alpha}\right)$ has no convergent subnet in $R$. If $\left\{x_{\alpha} \mid \alpha \in\right.$ I $\} \subset x_{1}$ then clearly $x \in D(g)$. If $\left\{x_{\alpha} \mid \alpha \in\right.$ I $\} \subset x-\left[x_{1} \cup D(f)\right]=D$, then $D \cup D(f)$ is nowhere dense in $X$. It can be shown as in Lemma 3.3 that there exists a net $\left\{x_{\beta} \mid \beta \in J\right\} \subset[D \cup D(f)]^{c} \subset x_{1}$ such that $x_{\beta} \rightarrow x$ and $f\left(x_{\beta}\right)$ has no convergent subnet in $R_{\text {. Since }} f\left(x_{\beta}\right)=g\left(x_{\beta}\right)$ for all $\beta \in J$, it follows that $x \in \mathrm{D}(\mathrm{g})$.

4. $X$ IS COMPLETELY REgULAR. Let $C(X)$ and $G(X)$ denote the families of all real valued functions on $X$ which are continuous and which have a closed graph, respectively. Let $\varphi(X)$ denote the family of all subsets of $X$. If $A \subset X$, let 
$|A|$ denote the cardinality of A. The next example shows that Theorem 3.2 does not hold for completely regular spaces.

EXAMPLE 4.1: Let $(X, \tau)$ denote the upper half of the euclidean plane $(y>0)$ with the tangent disc topology. Namely, put $P=\left\{(x, y) \in R^{2}|y\rangle 0\right\}$. Let $L=\left\{(x, y) \in R^{2} \mid y=0\right\}$. Put $X=P \cup L$. Let $\tau$ be the topology on $X$ such that $\tau$ restricted to $P$ is the usual euclidean topology. If $x \in L$ and $D$ is any open disc in $P$ tangent to $L$ at $x$, then $\{x\} \cup D$ is an open set in $x$ containing $x$ (Stein and Seeback [6], page 100). (X, $\tau)$ is completely regular and $L$ is closed and nowhere dense in $(X, \tau) \cdot|\varphi(L)|=2^{c}$, where $c$ is the cardinality of the continuum, and each $F \in P(L)$ is closed and nowhere dense in $X$. Let $f, g \in G(X)$ such that $D(f) \subset L, D(g) \subset L$ and $D(f) \neq D(g)$. Since $f \mid(X-L)$ and $g \mid(X-L)$ are both continuous, it follows from Lemma 3.4 that $f|(X-L) \neq g|(X-L)$. Since $|\mathrm{C}(\mathrm{X}-\mathrm{L})|=\mathrm{c}$, it follows that if $\mu \subset \mathcal{G}(\mathrm{X})$ has that property that for every $M \in \mathscr{H}$ there exists $f \in G(X)$ such that $D(f)=M$, then $|d| \leq c$. Therefore, there exists a family $\mathscr{F} \subset \mathscr{G}(\mathrm{L})$ such that $|\mathscr{F}|=|\mathscr{F}(\mathrm{L})|$ and for every $F \in F$ there does not exist a function $f \in G(X)$ such that $D(f)=F$.

REMARK 4.2: Let $\mathrm{X}$ be a topological space and let $\mathrm{L}$ be a closed and nowhere dense subset of $X$ which is relatively discrete as a subspace of $X$. If $|\rho(L)|>|C(X-L)|$, it follows from Lemma 3.4 that there exists a family $F$ of elements of $\mathscr{P}(\mathrm{L})$ such that $|\mathscr{F}|=|\mathscr{T}(\mathrm{L})|$ and for every $F \in \mathcal{F}$ there does not exist a function $f \in G(X)$ such that $D(f)=F$.

The following results hold when $X$ is completely regular.

THEOREM 4.3. Let $X$ be a completely regular space and let $F$ be a compact $G_{\delta}$ subset of $X$. There exists a function $f: X+R$ with a closed graph such that $D(f)=F$ if and only if $F$ is of first category in $X$.

PROOF: The necessity follows from Theorem 2.1. To show sufficiency, note that if $U$ is any open subset of $F, U$ is of first category since $F$ is of first category. $U$ is of second category since $F$ is compact and hence a Baire space. Therefore $U=\emptyset$ and $F$ is nowhere dense in $X$. Since $F$ is compact $G_{\delta}$, there exists, by Lemma 2.4 a continuous function $h: X+R$ such that $h^{-1}(0)=F$. Let $g: R+R$ be defined by $g(x)=\frac{1}{x}, x \neq 0$, and $g(0)=0$. By Proposition 2.3, if $f=g \circ h$, then $f$ has a closed graph. It follows that $D(f)=F$.

5. $X$ IS REGULAR. It follows from Example 4.1 that the condition 'let $F$ be compact' cannot be omitted in Theorem 4.3. Example I.6.1 by Hamlett and Herrington [3] shows that Theorem 4.3 does not hold if $F$ is not $G_{\delta}$. It will be shown in Example 5.2 that Theorem 4.3 does not hold in general for regular spaces. The topology on the space presented in Example 5.2 will be a refinement of the topology on a nice example of a regular space which is not completely regular that was constructed by Thomas [7]. An out1ine of Thomas' example follows. See [7] for further details and a geometric interpretation.

EXAMPLE 5.1 (THOMAS): If $n=0, \pm 1, \pm 2, \pm 3, \ldots$, put $\mathrm{L}(2 \mathrm{n})=$ $\left\{(2 n, y) \in R^{2} \mid 0 \leq y<\frac{1}{2}\right\}$. If $n=0, \pm 1, \pm 2, \pm 3, \ldots$, and $k=2,3,4, \ldots$, put $p(2 n-1, k)=\left(2 n-1,1-\frac{1}{k}\right) \epsilon R^{2}$, and put $T(2 n-1, k)=\left\{\left(2 n-1 \pm t, 1-t-\frac{1}{k}\right) \epsilon\right.$ $\left.\mathrm{R}^{2} \mid t \in\left(0,1-\frac{1}{k}\right]\right\}$. Let $a$ and $b$ be two points at 'infinity'. Put 


$$
X=\left\{\bigcup_{n=-\infty}^{+\infty} L(2 n)\right\} \cup\left\{\bigcup_{n=-\infty}^{\infty} \bigcup_{k=2}^{\infty}\{T(2 n-1, k) \cup p(2 n-1, k)]\right\} \cup\{a, b\} .
$$

Topologize $X$ as follows. If $x \in T(2 n-1, k)$ for $n=0, \pm 1, \pm 2, \ldots$ and $k=2,3, \ldots$, then $x$ is open. If $x=p(2 n-1, k)$, then a neighbourhood of $x$ contains all but finitely many points of $T(2 n-1, k)$. If $x=(2 n, y) \epsilon L(2 n)$, then a neighbourhood of $x$ consists of all but finitely many points of $x$ with the same $y$-coordinate and with $x$-coordinate that differs from $2 n$ by less than 1 . If $x=a$ and $c$ is a real number, then a subset of $X$ which consists of all points with $y$-coordinate $>c$ is an open set containing a. If $x=b$ and $c$ is a real number, then a subset of $X$ which consists of all points with $y$-coordinate < $c$ is an open set containing b. It was shown by Thomas [7] that $X$ is regular and if $f: X+R$ is continuous, then $f(a)=f(b)$.

Let $(X, \tau)$ be the space of Example 5.1. In the following example a topology $\tau^{\prime} \supset \tau$ will be constructed on $X$ such that $\left(X, \tau^{\prime}\right)$ is regular but not completely regular. For the space $\left(X, \tau^{\prime}\right)$ there will exist an $x \in X$ such that $\{x\}$ is $G_{\delta}$ but there will not exist a function $f \in G(X)$ such that $D(f)=\{x\}$.

EXAMPLE 5.2: Let $(X, \tau)$ be the space of Example 5.1. If $x \in X$ and $x \neq a$, let the neighbourhood base at $x$ be as defined in Example 5.1. Let $x=a$ and let $A=\left\{x_{1} \mid 1=1,2, \ldots\right\}$, where $x_{1} \neq$ a for all 1 , be a sequence in $x$ which converges to a in $(X, \tau)$. If for fixed $1, x_{1}$ is discrete in $(X, \tau)$, then put $s_{1}=\left\{x_{i}\right\}$. If for fixed $i, x_{1}=p(2 n-1, k)$ for some $n$ and $k$, put $s_{1}=\left\{x_{i}\right\} \cup T(2 n-1, k)$. If $x_{i}=\left(2 n, y_{0}\right) \in L(2 n)$ for some 1 and $n$, then put $S_{1}=\left\{\left(x, y_{0}\right) \in x \mid 2 n-1<x<2 n+1\right\}$. For each basic open neighbourhood $U$ of a in $(X, \tau)$ and each sequence $A$ converging to a in $(X, \tau)$ define $U-\left\{\underset{1=1}{U} S_{1}\right\}$ to be an open set containing a. Let $\tau$ ' be the topology on $X$ generated by $\tau$ and by the new neighbourhood base of a. Clearly $\tau^{\prime} \supset \tau$ and every open set $V$ in $\tau^{\prime}$ containing a contalns $T(2 n-1, k) \cup p(2 n-1, k)$ for countably many $n$ and k. Also $V$ contains all but countably many elements of $U$ to(2n). It follows from the construction that $\left(X, \tau^{\prime}\right)$ is regular. $\left(X, \tau^{\prime}\right)$ is not completely regular since it can be shown as in [7], that if $f:\left(X, \tau^{\prime}\right) \rightarrow R$ is continuous, $f(a)=f(b)$. \{a\} is $G_{\delta}$. There does not exist a function $g:\left(X, \tau^{\prime}\right)+R$ with $G(g)$ closed such that $D(g)=\{a\}$. For let $g:\left(X, \tau^{\prime}\right)+R$ be any function with a closed graph which is continuous on $X-\{a\}$. It can be shown as by Thomas [7] that $g(x)=c$, a constant, for all but countably many elements of $X-\{a\}$. Since a is a cluster point of $\left(X, \tau^{\prime}\right)$ but not of any sequence in $\left(X, \tau^{\prime}\right)$, it follows that any net $x_{\alpha}$ in $x$ which converges to $a$ in $\tau^{\prime}$ has the property that $g\left(x_{\alpha}\right) \rightarrow c$. Since the graph of $g$ is closed it follows that $g(a)=c$ and $g$ is continuous at a. Therefore Theorem 4.3 does not hold when $X$ is regular.

6. X IS A URYSOHN SPACE. In this section it will be shown that there exists a countable Urysohn space $X$ (Example 6.3) with the property that if $F$ is any non-empty, closed and nowhere dense subset of $x$, there does not exist a function $f: X \rightarrow R$ such that $G(f)$ is closed and $D(f)=F$ (Proposition 6.6). The space $X$ will be essentially the countable connected Urysohn space constructed by Roy [8]. 
The following proposition will be useful later:

PROPOSITION 6.1. Let $G$ be an open subset of a topological space $X$. If $G=\bigcup_{n=2}^{\infty} F_{n}$, where the $F_{n}$ 's are mutually disjoint, closed and nowhere dense in $X$, then there exists a function $f: X+R$ with $G(f)$ closed and $D(f)=\bar{G}$.

PROOF: Let $F_{0}=X-\bar{G}$ and $F_{1}=\bar{G}-G$. Define $f: X \rightarrow R$ by $f\left(F_{n}\right)=n$, for $n$ $=0,1,2, \ldots$ Since $f$ is constant on $X-\bar{G}, f$ is continuous on $X-\bar{G}$. Let $x \in \bar{G}$. Since for $n=1,2,3, \ldots$ each $F_{n}$ is closed and nowhere dense in $x$, there exists a net $\left\{x_{\alpha} \mid \alpha \in I\right\}$ in $G$ such that $x_{\alpha}+x$ and $F_{n}$ does not contain a subnet of $\left\{x_{\alpha} \mid \alpha \in I\right\}$ for each $n$. $\left\{x_{\alpha} \mid \alpha \in I\right\} \cap F_{n} \neq \emptyset$ for infinitely many $n^{\prime} s,\left\{f\left(x_{\alpha}\right) \mid \alpha \in I\right\}$ is unbounded and does not converge in $R$. Therefore $f$ is not continuous at $x$ and $D(f)=\bar{G}$. For each $x \in X$ and for each net $\left\{x_{\alpha}\right\}$ in $X$ which converges to $x$, it is easily seen that either $f\left(x_{\alpha}\right)+f(x)$ or $\left\{f\left(x_{\alpha}\right) \mid \alpha \in I\right\}$ has no convergence subnet in $R$. Therefore $G(f)$ is closed.

COROLLARY 6.2. Let $X$ be a countable connected $T_{2}$ space. If $G$ is an open subset of $X$, then there exists a function $f: X+R$ such that $G(f)$ is closed and $D(f)=\bar{G}$.

PROOF: Follows immediately from the preceding proposition.

EXAMPLE 6.3: Let $\left\{C_{n}\right\}_{n=-\infty}^{n=+\infty}$ be a countable collection of pairwise disjoint subsets of the rational numbers indexed by the set of positive and negative integers such that $C_{n}$ is dense in $R$ for each $n$. Let $x=\left\{(x, n) \mid x \in C_{n}\right.$, $\mathrm{n}=0, \pm 1, \pm 2, \ldots\} \cup\{\omega\}$. We may visualize $\mathrm{X}$ as beling 'lines' in the plane through $y=0, \pm 1, \pm 2, \pm 3, \ldots$, together with a point at 'infinity', w. For each $p \in X$, define a neighbourhood system for $p$ as follows. Let $p=(s, n) \epsilon X$ and let $\varepsilon>0$. If $n$ is even or zero, put $N_{\varepsilon}(p)=\left\{(x, y) \epsilon x \mid \dot{y}=n, x \in c_{y}\right.$ and $s-\varepsilon<x<s+\varepsilon\}$. If $n$ is odd put $N_{\varepsilon}(p)=\{(x, y) \in x \mid y=n, n+1$ or $n-1$, $x \in C_{y}$ and $\left.s-\varepsilon<x<s+\varepsilon\right\}$. If $p=w$, put $N_{\varepsilon}(p)=\left\{(x, y) \epsilon x \mid y>\frac{1}{\varepsilon}\right\}$. Let $\tau$ be the topology generated on $X$ by the nelghbourhood system defined above for all $\varepsilon>0 .(X, \tau)$ is essentially the space constructed by P. Roy $[8] \cdot(X, \tau)$ is a countable connected Urysohn space.

Let $F$ be a closed and nowhere dense subset of $X$. It will be established in Proposition 6.6 that if there exists a function $f: X+R$ such that $D(f)=F$, then $F=\emptyset$. Note that since $F$ is nowhere dense in $X, F \cap C_{2 n}$ is a nowhere dense subset of $I \cap C_{2 n}$ for each integer $n$ and every interval $I \subset R$. Also, for each $n, C_{2 n-1}$ is closed and nowhere dense in $X$. In what follows $v_{\varepsilon}(f(x))$ denotes an open neighbourhood of $f(x)$ of radius $\varepsilon$ in $R$.

PROPOSITION 6.4. Let $(X, \tau)$ be the space of Example 6.3, let $F \subset X$ be closed and nowhere dense and let $f: X+R$ be a function with $G(f)$ closed. If $D(f)=F$, then for every integer $n$ and for every open interval $I \subset R, f$ restricted to the subspace $I \cap \mathrm{C}_{2 n-1}$ is discontinuous on at most a nowhere dense subset of $I \cap C_{2 n-1}$ with the relative topology.

PROOF: Suppose on the contrary that for some $n$ and some open interval $I_{1} \subset R, f \mid\left(I_{1} \cap C_{2 n-1}\right)$ is discontinuous on a dense subset of the subspace 
$I_{1} \cap C_{2 n-1}$. Since $D(f)$ is nowhere dense in $x$, we may select $x \in I_{1} \cap C_{2 n}$ such that $f$ is continuous at $x$. Let $\varepsilon>0$, choose $\delta>0$ such that $N_{\delta}(x) \subset\left(I_{1} \cap C_{2 n}\right)$ and $f\left(N_{\delta}(x)\right) \subset v_{\varepsilon}(f(x))$. Select $s \in I_{1} \cap C_{2 n-1}$ such that $f \mid I_{1} \cap C_{2 n-1}$ is discontinuous at $s$ and such that every neighbourhood $U(s)$ of $s$ in $(X, \tau)$ has the property that $U(s) \cap C_{2 n} \subset N_{\delta}(x)$ for sufficiently small neighbourhoods of s. Since $f \mid\left(I_{1} \cap C_{2 n-1}\right)$ is discontinuous at 8 , there exists a sequence $\left\{s_{k} \mid k=1,2, \ldots\right\}$ such that $s_{k} \in I_{1} \cap C_{2 n-1}$ for all $k, s_{k}+s$, and, since $G(f)$ is closed, $\left\{f\left(s_{k}\right) \mid k=1,2, \ldots\right\}$ has no limit point in $R$. Select $s_{m} \in\left\{s_{k} \mid k=1,2, \ldots\right\}$ such that $f\left(s_{m}\right) \& v_{\varepsilon}(f(x))$. Then there exists a sequence $\left\{x_{n} \mid n=1,2, \ldots\right\} \subset N_{\delta}(x)$ where $x_{n}+s_{m}$ and $\left\{f\left(x_{n}\right) \mid n=1,2, \ldots\right\}$ converges to a point $p \in V_{\varepsilon}(f(x))$. Clearly $p \neq f\left(s_{m}\right)$. This contradicts the fact that the graph of $f$ is closed. Therefore $f \mid\left(I \cap C_{2 n-1}\right)$ is discontinuous on at most a nowhere dense subset of $I \cap C_{2 n-1}$ for every open interval $I \subset R$ and every integer $n$.

PROPOSITION 6.5. Let $(X, \tau)$ be the space of Example 6.3, let $F \subset X$ be closed and nowhere dense and let $f: X+R$ be a function with $G(f)$ closed. If $D(f)=F$, then for each 1nteger $n$ and for each open interval $I \subset R$ there exists a subinterval $I_{2 n-1} \subset I$ such that $f$ is continuous at $x$ for each $x \in I_{2 n-1} \cap C_{2 n-1}$

PROOF: Let $I$ be an open interval in $R$. For each integer $n$, there exists, by Proposition 6.4, an open interval $I_{2 n-1} \subset I$ such that $f \mid\left(I_{2 n-1} \cap C_{2 n-1}\right)$ is continuous on the subspace $I_{2 n-1} \cap C_{2 n-1}$ with the relative topology. Since $F$ is nowhere dense in $I \cap C_{2 n}$ and $I \cap C_{2 n-2}$ for each integer $n$, we may assume without $10 s 8$ of generality that $I_{2 n-1}$ has been chosen for each $n$ such that $f$ is continuous at each point of $\left(I_{2 n-1} \cap C_{2 n}\right) \cup\left(I_{2 n-1} \cap C_{2 n-2}\right)$.

Let $x \in I_{2 n-1} \cap C_{2 n-1}$ and 1 et $\left\{x_{n} \mid n=1,2, \ldots\right\}$ be a sequence in $x$ which converges to $x$. We may assume without loss of generality that $\left\{x_{n} \mid n=1,2, \ldots\right\}$ is eventually in either $I_{2 n-1} \cap C_{2 n-1}$ or $I_{2 n-1} \cap C_{2 n}$ or $I_{2 n-1} \cap C_{2 n-2}$. If $\left\{x_{n} \mid n=1,2, \ldots\right\}$ is eventually in $I_{2 n-1} \cap C_{2 n-1}$ then it follows from the way $I_{2 n-1}$ was selected that $f\left(x_{n}\right) \rightarrow f(x)$. Suppose $\left\{x_{n} \mid n=1,2, \ldots\right\}$ is eventually in $I_{2 n-1} \cap C_{2 n}$. Let $\varepsilon>0$. Since $f$ is continuous on $I_{2 n-1} \cap C_{2 n}$, for each $y \in I_{2 n-1} \cap C_{2 n}$ there exists a $\delta>0$ such that $f\left(N_{\delta}(y)\right) \subset V_{\varepsilon}(f(y))$. Select some $y \in I_{2 n-1} \cap C_{2 n}$ such that for all sufficiently small neighbourhoods $N(x)$ of $x$, $N(x) \cap C_{2 n} \subset N_{\delta}(y) \cdot \quad\left\{x_{n} \mid n=1,2, \ldots\right\}$ will eventually be in $N_{\delta}(y)$. Since $f\left(N_{\delta}(y)\right) \subset v_{\varepsilon}(f(y))$, it follows that $\left\{f\left(x_{n}\right) \mid n=1,2, \ldots\right\}$ is eventually in $v_{\varepsilon}(f(y))$ and we may assume that $\left\{f\left(x_{n}\right) \mid n=1,2, \ldots\right\}$ converges. Since the graph of $f$ is closed $f\left(x_{n}\right)+f(x)$. Similarly it follows that if $\left\{x_{n} \mid n=1,2, \ldots\right\}$ is eventually in $I_{2 n-1} \cap C_{2 n-2}, f\left(x_{n}\right)+f(x)$. Therefore $f$ is continuous at $x$ for each $x \in I_{2 n-1} \cap C_{2 n-1}$.

PROPOSITION 6.6. Let $(X, \tau)$ be the space of Example 6.3, let $F \subset X$ be closed and nowhere dense and let $f: X+R$ be a function with $G(f)$ closed. If $D(f)=F$, then $F=\emptyset$.

PROOF: Suppose $F$ is a non-empty, closed and nowhere dense subset of $X$ and that there exists a function $f: X \rightarrow R$ with $G(f)$ closed such that $D(f)=F$. If 
$f$ is constant on $X-F$, it follows that $f$ is constant on $X$ and hence continuous on $X$. We may assume therefore that $f$ is not constant on $X-F$. Let $y \in X-F$ and let $\varepsilon>0$ such that $f(\omega) \notin \overline{V_{\varepsilon}(f(y))}$. Since $f$ is continuous at $y$ there exists a $\delta>0$ and a neighbourhood $N_{\delta}(y)$ of $y$ such that $f\left(N_{\delta}(y)\right) \subset V_{\varepsilon}(f(y))$. We may assume without loss of generality that $N_{\delta}(y) \subset\left(I \cap C_{2 n-1}\right) \cup\left(I \cap C_{2 n}\right) \cup\left(I \cap C_{2 n-2}\right)$ for some interval $I \subset R$ and some integer $n$. It now follows from repeated applications of Proposition 6.5 and the definition of open sets in $(X, \tau)$ that for each $m>2 n$, there exists an interval $I_{m} \subset I$ such that $f\left(I_{m} \cap C_{m}\right) \subset v_{\varepsilon}(f(y))$. For each $m>2 n$, select $y_{m} \in\left(I_{m} \cap C_{m}\right)$. Then $y_{m}+\omega$ and $f\left(y_{m}\right) \in V_{\varepsilon}(f(y))$, for $m=2 n+1,2 n+2, \ldots$ Since $f(w) \notin \overline{v_{\varepsilon}(f(y))}$ this would contradict the fact that the graph of $f$ is closed. The proposition follows.

In contrast to the results of Dobðs [2] and Thompson [1] for perfectly normal spaces, it now follows that if $X$ satisfies only the Urysohn separation axiom it is possible that there may exist a function $f: X+R$ with a closed graph such that $D(f)=F$ if and only if $F$ is a closed subset of $X$ with a non-empty interior. That this is true for the space given in Example 6.3 follows immediately from Proposition 6.6 and Corollary 6.2.

REMARK 6.7: Proposition 6.6 does not hold for all countable connected Urysohn spaces although it does hold for the countable connected spaces constructed by Kannan [9] and Martin [10]. It is possible to redefine the topology on the space in Example 6.3 such that the space remains connected and Urysohn and $F=C_{1} \cup C_{0} \cup C_{-1}$ is closed and nowhere dense and there does exist a function $f: X \rightarrow R$ such that $G(f)$ is closed and $D(f)=F$.

REFERENCES

1. THOMPSON, T. Characterizing Certain Sets with Functions Having a Closed Graph, Bo11. del U.M.I. (4) 12(1975), 327-329.

2. DOBరS, J. On the Set of Points of Discontinuity for Functions with Closed Graphs, Cas. Pěst. Mat. 110(1985), 60-68.

3. HAMLETT, T.R. and HERRINGTON, L.L. The Closed Graph and P-Closed Graph Properties in General Topology, Vol. 3, Contemporary Mathematics, Amer. Math. Soc., Providence, Rhode Island, 1980.

4. GILLMAN, L. and JERISON, M. Rings of Continuous Functions, Van Nostrand, Princeton, 1960.

5. KURATOWSKI, C. Topology, Vo1. I (trans1.), Academic Press, New York, 1966.

6. STEIN, L.A. and SEEBACK, J.A. Counterexamples in Topology, Holt, Rinehart and Winston, Inc., New York, 1970.

7. THOMAS, J. A Regular Space, not Completely Regular, Amer. Math. Month1y, 76, (1969), 181-182.

8. ROY, P. A Countable Connected Urysohn Space with a Dispersion Point, Duke Math. J., 33(1966), 331-333.

9. KANNAN, V. A Countable Connected Urysohn Space Containing a Dispersion Point, Proc. Amer. Math. Soc., 35(1972), 289-290.

10. MARTIN, J. A Countable Hausdorff Space with a Dispersion Point, Duke Math. J., 33(1966), 165-167. 


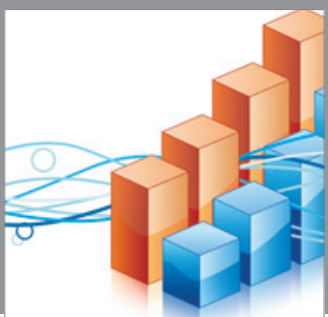

Advances in

Operations Research

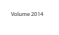

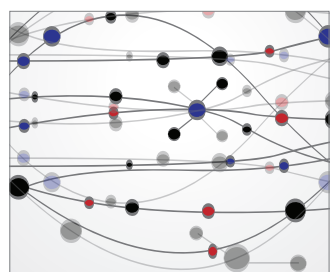

\section{The Scientific} World Journal
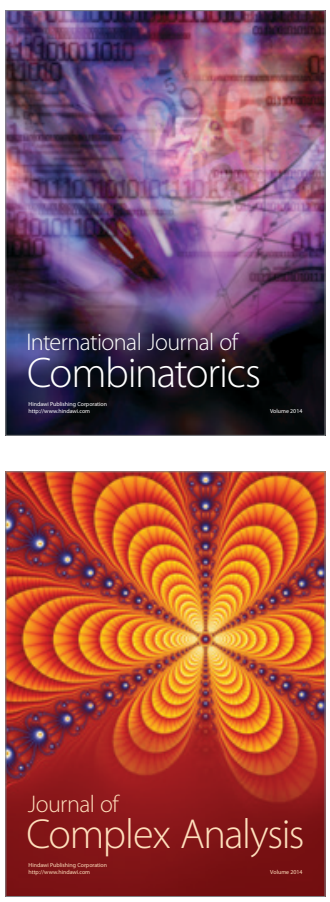

International Journal of

Mathematics and

Mathematical

Sciences
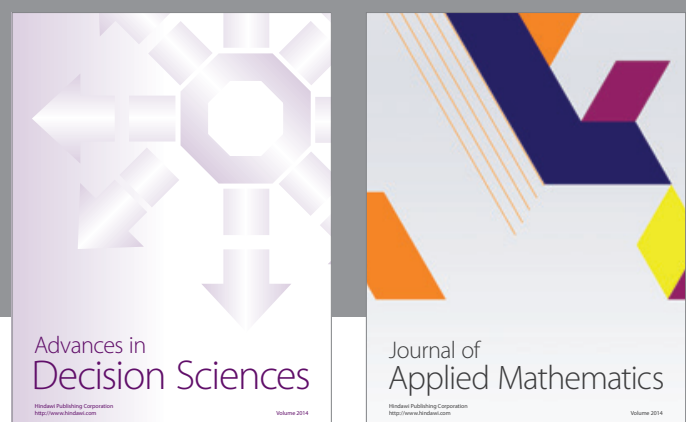

Journal of

Applied Mathematics
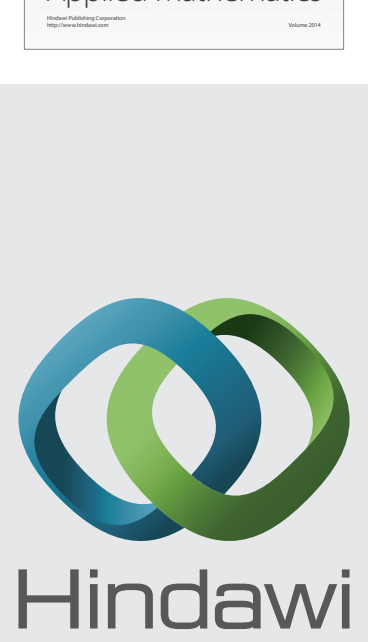

Submit your manuscripts at http://www.hindawi.com
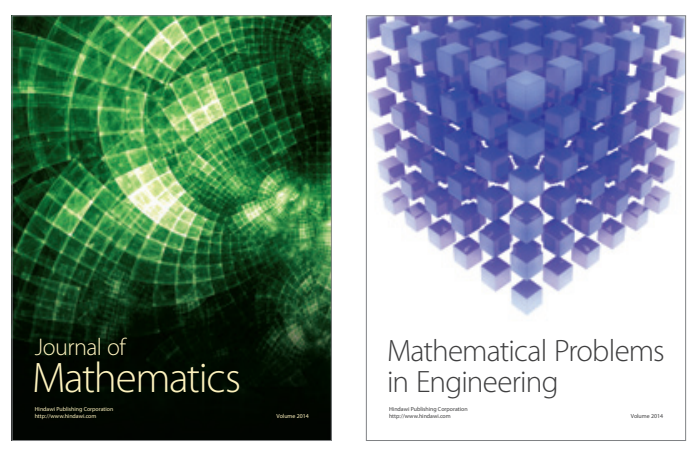

Mathematical Problems in Engineering
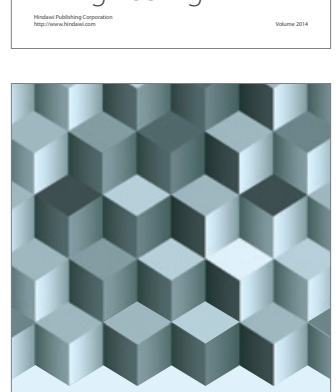

Journal of

Function Spaces
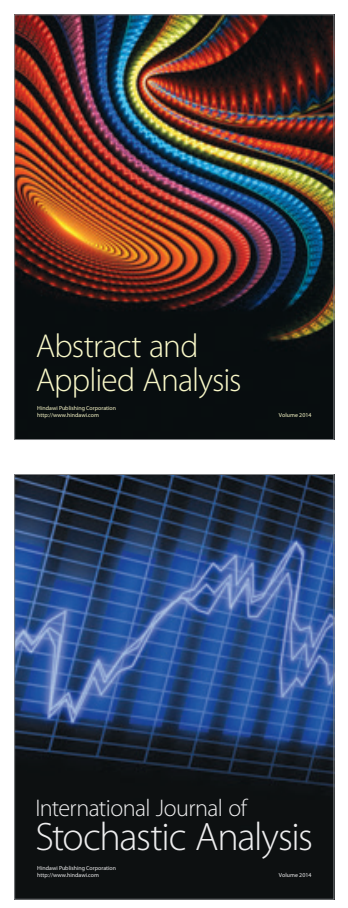

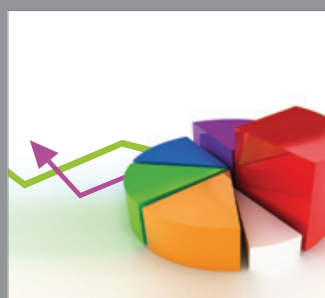

ournal of

Probability and Statistics

Promensencen
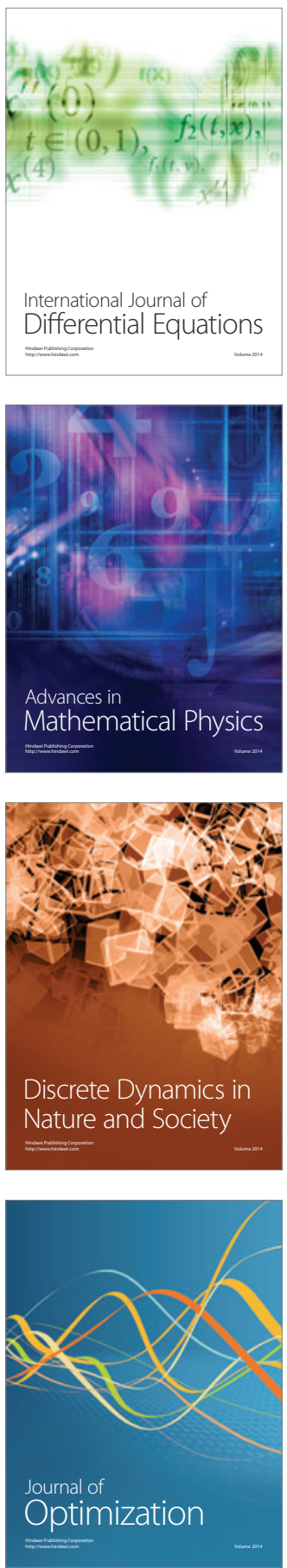